\title{
Endarterectomía carotídea con medición de presión de muñón, manejo hemodinámico y uso selectivo de shunt
}

\author{
Juan Bombin ${ }^{1,2}$, Alejandro Kotlik ${ }^{1,2}$, Gregory Córdova ${ }^{2}$, \\ Constanza Gómez ${ }^{2}$ y Maritchu Bombin ${ }^{3}$
}

\section{Carotid Endarterectomy with routine carotid stump pressure measurement, intraoperative hemodynamic management and selective use of shunt}

Introduction: During carotid endarterectomy (CEA) clamping cerebral perfusion is maintained by contralateral circulation through the Circle of Willis and it is correlated to the stump pressure (SP). If it is below $50 \mathrm{mmHg}$ there is risk of stroke due to hypoperfusion and a shunt must be used, but systemic blood pressure can be temporarily elevated making the use of shunt unnecessary. Aim: Results of CEA with SP measurement to evaluate cerebral perfusion in cross-clamped hemisphere and hemodynamic intraoperative management reducing the use of shunt. Material and Methods: Retrospective study of CEAs performed in 73 patients under general anaesthesia with SP measurement, hemodynamic management and selective use of shunt. Demographics, clinical and perioperative morbimortality variables were analized. Results: 73 patients, average age 71.1 years, 69.9\% symptomatic. In 54 patients SP was above $50 \mathrm{mmHg}$ and shunt was not used, in $19 \mathrm{SP}$ was below $50 \mathrm{mmHg}$, it was elevated through intraoperative hemodynamic management and shunt was not needed. In only 3 cases SP did not reach $50 \mathrm{mmHg}$ and a Pruitt-Inahara shunt was used. Two patients presented postoperative transient central neurological deficit and 2 died due to myocardial infarction. Conclusion: CEA with SP measurement and hemodynamic management reduced the use of carotid shunting and it was a safe procedure to treat patients with severe carotid stenosis who need surgical intervention.

Key words: Carotid endarterectomy; Stump pressure; Selective use of carotid shunt.

\section{Resumen}

Introducción: En la endarterectomía carotídea (EC) durante el clampeo, la perfusión cerebral se mantiene por circulación contralateral a través del Polígono de Willis, que se relaciona con la presión de muñón carotídeo (PM). Si ésta es menor a $50 \mathrm{mmHg}$ existe riesgo de Accidente Cerebrovascular (ACV) por hipoperfusión y está indicado uso de shunt para asegurar suficiente circulación cerebral, pero también se puede elevar transitoriamente la presión arterial sistémica haciendo innecesario el uso de shunt. Objetivo: Mostrar los resultados de EC con medición de PM para evaluar la perfusión cerebral del hemisferio clampeado con manejo hemodinámico intraoperatorio minimizando el uso de shunt. Material y Métodos: Estudio retrospectivo de 73 pacientes sometidos a EC bajo anestesia general con medición de PM, manejo hemodinámico intraoperatorio y uso selectivo de shunt. Se analizaron variables demográficas, clínicas y morbimortalidad perioperatoria. Resultados: 73 pacientes, edad promedio 71,1 años, 69,9\% sintomáticos. En 54 pacientes la PM fue superior a $50 \mathrm{mmHg}$ y no se usó shunt, en 19 la PM fue menor a $50 \mathrm{mmHg}$ y con manejo hemodinámico intraoperatorio se elevó en 16 que no requirieron shunt. Sólo en 3 casos la PM no alcanzó los $50 \mathrm{mmHg}$ y se usó un shunt de Pruitt-Inahara. Dos pacientes sintomáticos presentaron déficit neurológico central transitorio postoperatorio y 2 pacientes fallecieron por infarto cardíaco. Conclusión: La EC con medición de PM y manejo hemodinámico minimizó el uso de shunt transitorio y fue un procedimiento seguro para tratar los pacientes con estenosis carotídea con indicación quirúrgica. Palabras clave: Endarterectomía carotídea; Presión de muñón carotídeo; Uso selectivo de shunt carotídeo.
'Servicio de Cirugía. Hospital Dr. Eduardo Pereira. Valparaíso, Chile. ²Departamento de Cirugía. Universidad de Valparaíso. ${ }^{3}$ Unidad de Emergencia de Adultos. Hospital Carlos van Buren. Valparaíso, Chile.

Recibido el 15 de mayo de 2017, aceptado el 8 de agosto de 2017.

Correspondencia a: Juan Bombin F. drbombin@gmail.com 


\section{Introducción}

La endarterectomía carotídea (EC) sigue siendo el procedimiento quirúrgico de elección para tratar una estenosis carotídea significativa al restablecer el flujo cerebral normal pues extirpa la placa ateromatosa de la arteria carótida interna (ACI) afectada ${ }^{1-4}$, pero se requiere para ello un clampeo carotídeo transitorio. Durante ese momento la circulación de ese hemisferio cerebral depende de las arterias vertebrales y la arteria carótida contralateral a través del polígono de Willis. Para observar el grado de perfusión cerebral durante el clampeo se han desarrollado varios métodos, entre ellos, efectuar la cirugía con el paciente despierto bajo anestesia local o troncular observando los cambios neurológicos clínicos ${ }^{5,6}$. En pacientes sometidos a una anestesia general se puede monitorizar con un electroencefalograma (EEG) continuo ${ }^{7,8}$, potenciales evocados somatosensoriales ${ }^{9,10}$, doppler transcraneal ${ }^{11} \mathrm{u}$ otros medios sofisticados de alto costo. También se puede obtener información de la circulación cerebral midiendo en forma directa la presión arterial sistólica del muñón carotídeo, lo que se relaciona con la perfusión del hemisferio cerebral clampeado proveniente del lado contralateral ${ }^{12-14}$. Se ha reportado en la literatura que una presión arterial sistólica de muñón carotídeo menor a $50 \mathrm{mmHg}$ es indicación de la instalación selectiva de un shunt ${ }^{15}$. Actualmente existe la posibilidad de un manejo farmacológico que permite elevar la presión arterial sistémica y con ello la presión arterial del muñón carotídeo hasta niveles seguros, haciendo innecesario en la mayoría de los casos el uso de un shunt durante el clampeo.

Presentamos esta serie clínica de pacientes portadores de estenosis carotídea severa, intervenidos con una EC usando un shunt en forma selectiva, dependiendo de la medición de la presión sistólica de muñón carotídeo y manejo farmacológico de la presión sistémica durante el clampeo carotídeo.

\section{Material y Método}

Se trata de una serie clínica retrospectiva de 73 casos consecutivos de pacientes portadores de estenosis carotídea severa (mayor de $70 \%$ ) intervenidos con una endarterectomía carotídea (EC) durante 10 años desde el 2007 al 2016 en el Servicio de Cirugía del Hospital Dr. Eduardo Pereira de Valparaíso.

Se indicó esta cirugía en pacientes sintomáticos que habían sufrido un accidente cerebrovascular (ACV) con secuelas neurológicas no mayores en 26 casos, o uno o más episodios de crisis isquémicas transitorias en 25 pacientes. Se intervino también 22 pacientes asintomáticos que debían ser sometidos posteriormente a una cirugía de gran envergadura.

El grado de estenosis de la arteria carótida interna (ACI) fue evaluado mediante ecodoppler color en todos los pacientes y confirmado con Angioscanner de cayado aórtico, vasos cervicales y cerebrales para estudiar adecuadamente la anatomía vascular en 60 pacientes. En otros cuatro pacientes alérgicos a los medios de contraste yodados se realizó una angioresonancia de esos vasos.

Todas las intervenciones fueron efectuadas bajo anestesia general, con intubación endotraqueal, monitorización invasiva de la presión arterial sistémica y de presión venosa central, electrocardiografía continua y oximetría de pulso. Durante la cirugía los pacientes se mantuvieron en leve posición de Trendelenburg invertido para disminuir la presión venosa yugular y cerebral.

La técnica quirúrgica consistió en una disección cervical cuidadosa y exposición de la bifurcación carotídea, heparinización sistémica efectiva medida con uso de ACT (Activated clotting time) y posterior clampeo carotídeo para extraer la placa ateromatosa obstructiva. En ese momento se midió en todos los pacientes la presión arterial de muñón carotídeo (PM) mediante punción directa de la arteria carótida común con una braunula $18 \mathrm{Fr}$ conectada a una línea de presión arterial ${ }^{5}$. Según estableció Moore y colaboradores ${ }^{12}$, la PM se logra al clampear las arterias carótida común, externa y tiroídea superior, pero dejando libre el flujo arterial retrógrado por la ACI (Figura 1, adaptada de Moore et $\mathrm{al}^{12}$ ). La PM se correlaciona con la presión de perfusión del hemisferio cerebral clampeado ${ }^{6,12,16}$. Se consideró que una presión de muñón carotídeo de $50 \mathrm{~mm} \mathrm{Hg}$ o mayor es suficiente para tener una perfusión cerebral mínima sin daño neurológico, lo que ocurrió en 54 pacientes. La PM fue menor en 19 pacientes, pero se logró elevar mediante el uso racional y transitorio de fármacos vasoactivos durante el período de clampeo arterial en 16 casos. Con ese fin se usó una solución fisiológica con fenilefrina en bomba de infusión endovenosa, titulando la dosis para elevar la presión arterial sistólica sistémica (sin sobrepasar $160 \mathrm{~mm}$ $\mathrm{Hg}$ ), y consecuentemente la PM a $50 \mathrm{~mm} \mathrm{Hg}$, suspendiendo el medicamento al término del período del clampeo. Todos ellos fueron intervenidos sin shunt. En sólo tres casos la PM medida fue menor a $50 \mathrm{~mm} \mathrm{Hg}$ y se mantuvo a pesar del uso de drogas vasoactivas por lo que fue necesario el uso de un shunt transitorio de Pruitt Inahara para mantener la perfusión cerebral ${ }^{17}$.

La EC se realizó mediante una incisión longitu- 
Tabla 1. Endarterectomía carotídea con toma de PM, manejo hemodinámico y uso selectivo de shunt. Morbimortalidad postoperatoria a 30 días en 73 pacientes operados

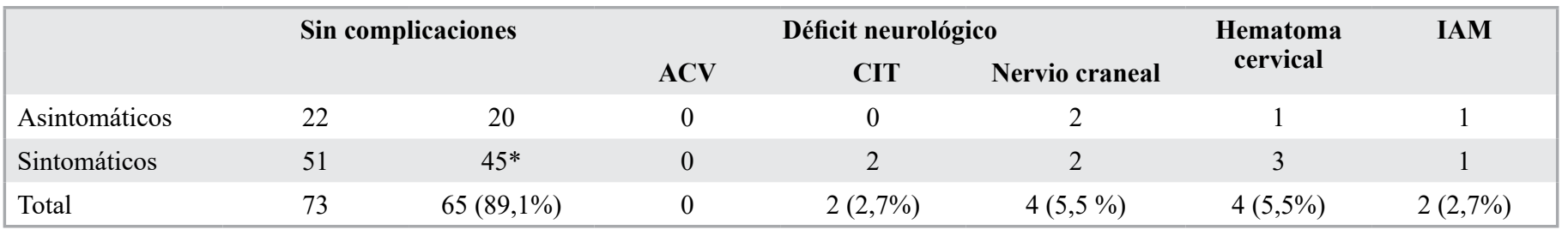

Diferencias entre los diversos grupos sin significación estadística. *Se incluyen los 3 pacientes en que se usó un shunt de Pruitt-Hinahara. PM: Presión de muñón carotídeo. ACV: Accidente cerebrovascular ("Stroke"). CIT: Crisis isquémica transitoria. IAM: Infarto agudo de miocardio.

dinal en la arteria carótida común extendiéndola a la ACI, cuidadosa extracción de la placa ateromatosa, fijación de la íntima distal y uso de parche en la mayoría de los casos. En el postoperatorio los pacientes fueron llevados a una Unidad de Paciente Crítico con monitorización estricta de la presión arterial. Todos fueron sometidos a una doble antiagregación plaquetaria con ácido acetilsalicílico y clopidogrel.

Se analizaron los datos demográficos, patología asociada, causa de consulta, uso de shunt, mortalidad y complicaciones del procedimiento hasta los 30 días del postoperatorio, los que fueron sometidos a pruebas de significación estadística.

\section{Resultados}

De la casuística de 73 pacientes hubo 49 hombres $(67,1 \%)$, con una edad promedio de 71,1 años (rango de 53 a 85 años). Fueron factores de riesgo prevalentes: hipertensión arterial $(94,3 \%)$, dislipidemia $(68,6 \%)$, diabetes mellitus $(44,3 \%)$, tabaquismo $(41,1 \%)$, coronariopatía $(30 \%)$ y ateromatosis periférica $(27,1 \%)$.

La patología carotídea fue diagnosticada en todos los pacientes mediante un Ecodoppler color y Angioscanner en 60 casos (82\%) o angioresonancia en $4(5,5 \%)$. En nueve pacientes la indicación quirúrgica se basó en el Ecodoppler color en forma exclusiva. En todos se realizó un scanner cerebral pre-operatorio. Todos los pacientes presentaron una estenosis de la arteria carótida interna de un 70\% o mayor. Fueron sintomáticos 51 pacientes $(69,8 \%)$, 26 de ellos sufrieron un accidente cerebrovascular (ACV) con secuelas moderadas o menores (51\%) y 25 pacientes presentaron una crisis isquémica transitoria (CIT) (49\%).

En un 92\% la arteriorrafia carotídea se realizó con parche. En 50 pacientes se usó un parche de poliuretano $(75 \%), 10$ pacientes con un trozo de vena safena interna proximal $(15 \%)$ y parche de politetrafluoretileno en 7 casos.
Sesenta y cinco pacientes $(89,1 \%)$ cursaron su postoperatorio sin presentar complicaciones. Entre los pacientes previamente sintomáticos que habían sufrido un ACV, hubo 2 pacientes con déficit neurológico postoperatorio transitorio sin mayores secuelas posteriores. Los tres pacientes en que se usó un shunt transitorio fueron sintomáticos. No hubo déficit neurológico central postoperatorio en los pacientes asintomáticos, ni en aquellos en que se usó un shunt transitorio. Cuatro pacientes presentaron déficit postoperatorio de algún nervio craneano, tres del nervio hipogloso y uno tuvo un déficit de la rama mandibular del nervio facial, todos transitorios y sin secuelas. Cuatro pacientes presentaron un hematoma cervical que no requirió intervención quirúrgica, con reabsorción espontánea. Dos pacientes fallecieron dentro de los 30 días de postoperatorio a causa de un infarto cardíaco, a los 13 y a los 29 días (Tabla 1). Estos resultados no demostraron diferencias estadísticamente significativas entre los diferentes grupos de pacientes.

\section{Discusión}

Existe una permanente controversia sobre el uso rutinario o selectivo de shunt como un medio para prevenir la hipoperfusión e isquemia cerebral durante el clampeo en una endarterectomía carotídea (EC). Existen varios métodos para evaluar la perfusión cerebral: se pueden observar los cambios neurológicos en un paciente despierto ${ }^{5,6}$, cambios en un electroencefalograma (EEG) continuo ${ }^{7,8,18}$, medición de potenciales evocados somatosensoriales ${ }^{9,10}$, uso de Doppler transcraneal ${ }^{11}$, medición de la oxigenación con espectroscopía infrarroja ${ }^{19} \mathrm{o}$ la medición de la presión arterial sistólica de muñón carotídeo $(\mathrm{PM})^{12-14}$. Algunos cirujanos prefieren uso rutinario de shunt para mantener la perfusión cerebral durante el clampeo carotídeo en pacientes bajo anestesia general $^{20}$, pero esta técnica puede estar asociada a 
daño intimal, disección de la arteria carótida interna o embolización aérea o de placas que pueden pasar incluso a través del shunt, provocando un accidente cerebrovascular (ACV) intraoperatorio ${ }^{7,21}$. Se ha reportado incluso que el riesgo adicional de $\mathrm{ACV}$, dado por el uso de un shunt, puede ser igual o mayor que el debido a isquemia hemodinámica ${ }^{7}$. En muchos lugares del mundo algunos cirujanos vasculares prefieren evaluar cualquier cambio neurológico durante la EC en un paciente despierto intervenido bajo anestesia local o troncular, dado que este método es muy sensible y precoz en caso de isquemia cerebral ${ }^{5,6}$, sin embargo, se ha comunicado que muchos pacientes no toleran la claustrofobia y ansiedad provocada por tener el rostro cubierto y la posición durante la cirugía, y por otra parte, no es conveniente en pacientes con cuello rígido por cervicopatías, cirugía en reintervenciones u otras razones técnicas.

Los pocos estudios científicos rigurosos y metaanálisis que compararon el uso rutinario de shunt versus no usar shunt no encontraron diferencias significativas en cuanto a accidentes cerebrovasculares o muertes dentro de 30 días postoperatorios ${ }^{22-24}$.

La evaluación de la perfusión cerebral durante el clampeo se realiza en la mayoría de los centros quirúrgicos del mundo mediante la observación de un trazado EEG continuo o midiendo la presión arterial sistólica de muñón carotídeo, métodos que tienen costos muy diferentes ${ }^{7,25}$. En el primer caso se requiere el equipamiento y un médico experto en la interpretación del trazado EEG, lo que es de alto costo y difícil de obtener en nuestro medio, y en el segundo caso sólo se debe contar con un equipo de punción y medición arterial directa, disponibles en la mayoría de los centros de nuestro país, con costos razonables.

Diversos estudios, usando monitorización continua EEG y medición de presión de muñón, han establecido que la presión sistólica de muñón mínima es entre 25 y $50 \mathrm{mmHg}$, bajo la cual habría isquemia cerebral, indicando en estos casos la colocación de un shunt transitorio ${ }^{12-15}$. La incidencia de cambios en el EEG fue sólo un 2\% cuando la presión de muñón fue de $50 \mathrm{~mm} \mathrm{Hg}$ o mayor ${ }^{8}$. Pero si la presión arterial de muñón es más baja que esa cifra, actualmente es posible elevar la presión sistémica y de muñón usando criteriosamente drogas vasoactivas en forma transitoria durante el período de clampeo de la arteria carótida, evitando de ese modo la instalación de un shunt, con la potencial morbilidad que implica y las incomodidades técnicas para el cirujano.

En esta serie clínica medimos rutinariamente la
PM. Si la PM estaba sobre $50 \mathrm{~mm} \mathrm{Hg}$, se procedió a la endarterectomía sin shunt, si la PM estaba bajo 50 $\mathrm{mm} \mathrm{Hg}$, se utilizó drogas vasoactivas para elevar y mantener la presión sistémica y la PM hasta obtener al menos $50 \mathrm{~mm} \mathrm{Hg}$ para efectuar la endarterectomía sin shunt. En sólo tres casos de esta serie no se logró subir la PM hasta niveles seguros, por lo que fue necesario el uso de un shunt. Se trató de pacientes con obstrucción o severa estenosis de la arteria carótida contralateral ${ }^{25}$.

\section{Conclusión}

Se desprende de nuestra experiencia que el uso rutinario de un shunt en la endarterectomía carotídea no está justificado. La medición de la perfusión cerebral a través de medición de la presión de muñón es un procedimiento sencillo y barato al alcance de la mayoría de los centros quirúrgicos de nuestro medio, y permite seleccionar en forma confiable a los pacientes para efectuar la endarterectomía carotídea sin uso de un shunt en la mayoría de los casos. El manejo hemodinámico de la presión sistémica permite elevar la presión del muñón, haciendo innecesario el uso de un shunt, con la sola excepción de aquellos que tienen una grave enfermedad ateromatosa carotídeo-vertebral bilateral.

\section{Responsabilidades éticas}

Protección de personas y animales. Los autores declaran que para esta investigación no se han realizado experimentos en seres humanos ni en animales.

Confidencialidad de los datos. Los autores declaran que han seguido los protocolos de su centro de trabajo sobre la publicación de datos de pacientes.

Derecho a la privacidad y consentimiento informado. Los autores declaran que en este artículo no aparecen datos de pacientes.

\section{Financiación}

No existe ningún interés ni soporte financiero o comercial en este manuscrito.

\section{Conflicto de intereses}

Los autores declaran no tener ningún conflicto de intereses. 


\section{Bibliografía}

1. North American Symptomatic Carotid Endarterectomy Trial Collaborators. Beneficial effect of carotid endarterectomy in symptomatic patients with highgrade carotid stenosis. N Engl J Med. 1991;325:445-53.

2. European Carotid Trialists' Collaborative Group. MRC European Carotid Surgery Trial: interim results for symptomatic patients with severe (70-99\%) or with mild $(0-29 \%)$ carotid stenosis. Lancet 1991;337:1235-43.

3. Moore WS, Barnett HJ, Beebe HG, Bernstein EF, Brener BJ, Brott T, et al. Guidelines for carotid endarterectomy: a multidisciplinary consensus statement from the Ad Hoc Committee, American Heart Association. Stroke 1995;26:188201. Review.

4. Executive Committee for the Asymptomatic Carotid Atherosclerosis Study Endarterectomy for asymptomatic carotid artery stenosis. JAMA 1995;273:1421-1.

5. Calligaro KD, Dougherty MJ. Correlation of carotid artery stump pressure and neurologic changes during 474 carotid endarterectomies performed in awake patients. J Vasc Surg. 2005;42:684-9.

6. Hans SS, Jarupoon O. Prospective evaluation of electroencephalography, carotid artery stump pressure, and neurologic changes during 314 consecutive carotid endarterectomies performed in awake patients. J Vasc Surg. 2007;45:511-5.

7. Green RM, Messick WJ, Ricotta JJ, Charlton MJ, Satran R, McBride MM, et al. Benefits, shortcoming and costs of EEG monitoring. Ann Surg. 1983;197:707-13.

8. Cherry KJ Jr, Roland CF, Hallett JW Jr, Gloviczki P, Bower TC, Toomey BJ, et al. Stump pressure, the contralateral carotid artery, and electroencephalographic changes. Am J Surg. 1991;162:185-9.

9. Haupt WF, Horsch S. Evoked potential monitoring in carotid surgery: a review of 994 cases. Neurology 1992;42:835-8.

10. Poblete R, Draper S, Velásquez A, Mellado L, Thieck E, Acuña R. Control de la circulación cerebral mediante el uso de Potenciales Evocados Somato-Sensitivos durante la Cirugía Carotídea. Rev Chil Cir. 1991;43:82-5.

11. McCarthy RJ, McCabe AE, Walker R, Horrocks M. The value of transcanial Doppler in predicting cerebral ischaemia during carotid endarterectomy. Eur J Vasc Endovasc Surg. 2001;21:408-12.

12. Moore WS, Hall AD. Carotid artery back pressure. Arch Surg. 1969;99:702.

13. Archie JP. Technique and clinical results of carotid stump back-pressure to determine selective shunting during carotid endarterectomy. J Vasc Surg. 1991;13:319-27.

14. Whitely D, Cherry KJ. Predictive value of carotid artery stump pressure during carotid endarterectomy. Neurosurg Clin NA. 1996;7:723-32.

15. Allai R, Marone LK, Melrzer J, Jevalaban G. Carotid endarterectomy. Int Anesthesiol Clin. 2005; 43:15-38

16. Harada RN, Comerota AJ, Good GM, Hashemi HA, Hulihan JF. Stump pressure, electroencephalografic changes, and the contralateral carotid artery: another look at selective shunting. Am J Surg. 1995; 170:148-53.
17. Hayes PD, Vainas T, Hartley S, Thompson NM, London NJ, Bell PR, Naylor AR. The Pruitt-Inahara shunt maintains mean middle cerebral artery velocities within $10 \%$ of preoperative values during carotid endarterectomy. J Vasc Surg. 2000; 32:299-306.

18. Fletcher JP, Morris JG, Little JM, Kershaw LZ. EEG monitoring during carotid endarterectomy. Aust N Z Surg. 1988;58:285-8.

19. Samra SK, Dorje P, Zelenock GB, Stanley JC. Cerebral oximetry in patients undergoing carotid endarterectomy under regional anesthesia. Stroke 1996;27:49-55.

20. Mertens R. Endarterectomía carotídea. Rev Chil Cir. 1997;49:130-7.

21. Howell SJ. Carotid endarterectomy. Br J Anaesth. 2007;99:119-3.

22. Bond R, Rerkasem K, Counsell C, Salinas R, Naylor R, Warlow CP, Rothwell PM. Routine or selective carotid artery shunting for carotid artery surgery (and different methods of monitoring in selective shunting). Cochrane Database Syst Rev 2002;(4):CD000190.

23. Sandmann W, Kolvenbach R, Willeke F. Risks and benefits of shunting in carotid endarterectomy. Stroke 1993;24:1098-9.

24. Gumerlock MK, Neuwelt EA. Carotid endarterectomy: to shunt or not shunt. Stroke 1988;19:1485-90.

25. Schneider JR, Droste JS, Schindler N, Golan JF, Berstein LP, Rosenberg RS, Carotid endarterectomy with routine electroencephalography and selective shunting: influence of contralateral internal carotid artery occlusion and utility in prevention of perioperative strokes. J Vasc Surg. 2002;35:1114-22. 